\title{
Application of the Local Fractional Series Expansion Method and the Variational Iteration Method to the Helmholtz Equation Involving Local Fractional Derivative Operators
}

\author{
Ai-Min Yang, ${ }^{1,2}$ Zeng-Shun Chen, ${ }^{3}$ H. M. Srivastava, ${ }^{4}$ and Xiao-Jun Yang ${ }^{5}$ \\ ${ }^{1}$ College of Science, Hebei United University, Tangshan 063009, China \\ ${ }^{2}$ College of Mechanical Engineering, Yanshan University, Qinhuangdao 066004, China \\ ${ }^{3}$ School of Civil Engineering and Architecture, Chongqing Jiaotong University, Chongqing 400074, China \\ ${ }^{4}$ Department of Mathematics and Statistics, University of Victoria, Victoria, BC, Canada V8W 3R4 \\ ${ }^{5}$ Department of Mathematics and Mechanics, China University of Mining and Technology, Jiangsu, Xuzhou 221008, China
}

Correspondence should be addressed to Ai-Min Yang; aimin_heut@163.com

Received 31 July 2013; Accepted 17 October 2013

Academic Editor: Bashir Ahmad

Copyright (C) 2013 Ai-Min Yang et al. This is an open access article distributed under the Creative Commons Attribution License, which permits unrestricted use, distribution, and reproduction in any medium, provided the original work is properly cited.

\begin{abstract}
We investigate solutions of the Helmholtz equation involving local fractional derivative operators. We make use of the series expansion method and the variational iteration method, which are based upon the local fractional derivative operators. The nondifferentiable solution of the problem is obtained by using these methods.
\end{abstract}

\section{Introduction}

The Helmholtz equation is known to arise in several physical problems such as electromagnetic radiation, seismology, and acoustics. It is a partial differential equation, which models the normal and nonfractal physical phenomena in both time and space [1]. It is an important differential equation, which is usually investigated by means of some analytical and numerical methods (see [2-11] and the references therein). For example, the FEM solution for the Helmholtz equation in one, two, and three dimensions was investigated in $[2,3]$. The variational iteration method was used to solve the Helmholtz equation in [4]. The explicit solution for the Helmholtz equation was considered in [5] by using the homotopy perturbation method. The domain decomposition method for the Helmholtz equation was presented in [6]. The boundary element method for the Helmholtz equation was considered in $[7,8]$. The modified Fourier-Galerkin method for the Helmholtz equations was applied in [9]. The Green's function for the two-dimensional Helmholtz equation in periodic domains was suggested in $[10,11]$.
Fractional calculus theory [12-26] has been applied to deal with the differentiable models from the practical engineering discipline, which are the anomalous and fractal physical phenomena. The fractional Helmholtz equations were considered in [27-29]. In this work, there are two methods to deal with such problems. For example, an analytic solution for the fractional Helmholtz equation in terms of the Mittag-Leffler function was investigated in [28]. The homotopy perturbation method for multidimensional fractional Helmholtz equation was considered in [29].

Local fractional calculus theory [30-44] has been used to process the nondifferentiable problems in natural phenomena. Taking an example, the local fractional Fokker-Planck equation was proposed in [30]. The mechanics of quasibrittle materials with a fractal microstructure with the local fractional derivative was presented in [31]. The anomalous diffusion modeling by fractal and fractional derivatives was considered in [35]. The local fractional wave and heat equations were discussed in $[36,37]$. Newtonian mechanics on fractals subset of real-line was investigated in [38]. In [39], the Helmholtz equation on the Cantor sets involving local 
fractional derivative operators was proposed. There are some other methods to handle the local fractional differential equations, such as local fractional series expansion method [40] and variational iteration method [41-44].

The main objective of the present paper is to solve the Helmholtz equation involving the local fractional derivative operators by means of the local fractional series expansion method and the variational iteration method. The structure of the paper is as follows. In Section 2, we describe the Helmholtz equation involving the local fractional derivative operators. In Section 3, we give analysis of the methods used. In Section 4, we apply the local fractional series expansion method to deal with the Helmholtz equation. In Section 5, we apply the local fractional variational iteration method to deal with the Helmholtz equation. Finally, in Section 6, we present our conclusions.

\section{Helmholtz Equations within Local Fractional Derivative Operators}

The Helmholtz equation involving local fractional derivative operators was proposed.

Let us denote the local fractional derivative as follows [36, 37, 39-44]:

$$
f^{(\alpha)}\left(x_{0}\right)=\left.\frac{d^{\alpha} f(x)}{d x^{\alpha}}\right|_{x=x_{0}}=\lim _{x \rightarrow x_{0}} \frac{\Delta^{\alpha}\left(f(x)-f\left(x_{0}\right)\right)}{\left(x-x_{0}\right)^{\alpha}},
$$

where $\Delta^{\alpha}\left(f(x)-f\left(x_{0}\right)\right) \cong \Gamma(1+\alpha) \Delta\left(f(x)-f\left(x_{0}\right)\right)$.

Using separation of variables in nondifferentiable functions, the three-dimensional Helmholtz equation involving local fractional derivative operators was suggested by the following expression [39]:

$$
\begin{aligned}
\frac{\partial^{2 \alpha} M(x, y, z)}{\partial x^{2 \alpha}} & +\frac{\partial^{2 \alpha} M(x, y, z)}{\partial y^{2 \alpha}}+\frac{\partial^{2 \alpha} M(x, y, z)}{\partial z^{2 \alpha}} \\
& +\omega^{2 \alpha} M(x, y, z)=0,
\end{aligned}
$$

where the operator involved is a local fractional derivative operator.

In this case, the two-dimensional Helmholtz equation involving local fractional derivative operators is expressed as follows (see [39]):

$$
\frac{\partial^{2 \alpha} M(x, y)}{\partial x^{2 \alpha}}+\frac{\partial^{2 \alpha} M(x, y)}{\partial y^{2 \alpha}}+\omega^{2 \alpha} M(x, y)=0
$$

The three-dimensional inhomogeneous Helmholtz equation is given by (see [39])

$$
\begin{aligned}
\frac{\partial^{2 \alpha} M(x, y, z)}{\partial x^{2 \alpha}} & +\frac{\partial^{2 \alpha} M(x, y, z)}{\partial y^{2 \alpha}}+\frac{\partial^{2 \alpha} M(x, y, z)}{\partial z^{2 \alpha}} \\
& +\omega^{2 \alpha} M(x, y, z)=f(x, y, z),
\end{aligned}
$$

where $f(x, y, z)$ is a local fractional continuous function.
The two-dimensional local fractional inhomogeneous Helmholtz equation is considered as follows (see [39]):

$$
\frac{\partial^{2 \alpha} M(x, y)}{\partial x^{2 \alpha}}+\frac{\partial^{2 \alpha} M(x, y)}{\partial y^{2 \alpha}}+\omega^{2 \alpha} M(x, y)=f(x, y) \text {, }
$$

where $f(x, y)$ is a local fractional continuous function.

The previous local fractional Helmholtz equations with local fractional derivative operators are applied to describe the governing equations in fractal electromagnetic radiation, seismology, and acoustics.

\section{Analysis of the Methods Used}

3.1. The Local Fractional Series Expansion Method. Let us consider a given local fractional differential equation

$$
u_{t}^{2 \alpha}=L_{\alpha} u,
$$

where $L$ is a linear local fractional derivative operator of order $2 \alpha$ with respect to $x$.

By the local fractional series expansion method [40], a multiterm separated function of independent variables $t$ and $x$ reads as

$$
u(x, t)=\sum_{i=0}^{\infty} T_{i}(t) X_{i}(x),
$$

where $T_{i}(t)$ and $X_{i}(x)$ are local fractional continuous functions.

In view of (7), we have

$$
T_{i}(t)=\frac{t^{i \alpha}}{\Gamma(1+i \alpha)},
$$

so that

$$
u(x, t)=\sum_{i=0}^{\infty} \frac{t^{i \alpha}}{\Gamma(1+i \alpha)} X_{i}(x)
$$

Making use of (9), we get

$$
\begin{gathered}
u_{t}^{2 \alpha}=\sum_{i=0}^{\infty} \frac{1}{\Gamma(1+i \alpha)} t^{i \alpha} X_{i+2}(x), \\
L_{\alpha} u=L_{\alpha}\left[\sum_{i=0}^{\infty} \frac{t^{i \alpha}}{\Gamma(1+i \alpha)} X_{i}(x)\right]=\sum_{i=0}^{\infty} \frac{t^{i \alpha}}{\Gamma(1+i \alpha)}\left(L_{\alpha} X_{i}\right)(x) .
\end{gathered}
$$

In view of (10), we have

$$
\sum_{i=0}^{\infty} \frac{1}{\Gamma(1+i \alpha)} t^{i \alpha} X_{i+2}(x)=\sum_{i=0}^{\infty} \frac{t^{i \alpha}}{\Gamma(1+i \alpha)}\left(L_{\alpha} X_{i}\right)(x) .
$$

Hence, from (11), the recursion reads as follows:

$$
X_{i+2}(x)=\left(L_{\alpha} X_{i}\right)(x) .
$$

By using (12), we arrive at the following result:

$$
u(x, t)=\sum_{i=0}^{\infty} \frac{t^{i \alpha}}{\Gamma(1+i \alpha)} X_{i}(x)
$$


3.2. The Local Fractional Variational Iteration Method. Let us consider the following local fractional operator equation:

$$
L_{\alpha} u+R_{\alpha} u=g(t)
$$

where $L_{\alpha}$ is linear local fractional derivative operator of order $2 \alpha, R_{\alpha}$ is a lower-order local fractional derivative operator, and $g(t)$ is the inhomogeneous source term.

By using the local fractional variational iteration method [41-44], we can construct a correctional local fractional functional as follows:

$$
\begin{aligned}
u_{n+1}(x)= & u_{n}(x)+{ }_{0} I_{x}^{(\alpha)} \\
& \times\left\{\eta(s)\left[L_{\alpha} u_{n}(s)+R_{\alpha} \tilde{u}_{n}(s)-\tilde{g}(s)\right]\right\},
\end{aligned}
$$

where the local fractional operator is defined as follows [36, 37, 41-44]:

$$
\begin{aligned}
a_{b}^{(\alpha)} f(x) & =\frac{1}{\Gamma(1+\alpha)} \int_{a}^{b} f(t)(d t)^{\alpha} \\
& =\frac{1}{\Gamma(1+\alpha)} \lim _{\Delta t \rightarrow 0} \sum_{j=0}^{j=N-1} f\left(t_{j}\right)\left(\Delta t_{j}\right)^{\alpha}
\end{aligned}
$$

and a partition of the interval $[a, b]$ is $\Delta t_{j}=t_{j+1}-t_{j}, \Delta t=$ $\max \left\{\Delta t_{1}, \Delta t_{2}, \Delta t_{j}, \ldots\right\}$, and $j=0, \ldots, N-1, t_{0}=a, t_{N}=b$.

Following (15), we have

$$
\begin{aligned}
\delta^{\alpha} u_{n+1}(x)= & \delta^{\alpha} u_{n}(x)+{ }_{0} I_{x}^{(\alpha)} \delta^{\alpha} \\
& \times\left\{\eta(s)\left[L_{\alpha} u_{n}(s)+R_{\alpha} \widetilde{u}_{n}(s)-\tilde{g}(s)\right]\right\} .
\end{aligned}
$$

The extremum condition of $u_{n+1}$ is given by $[37,41,42]$

$$
\delta^{\alpha} u_{n+1}=0 \text {. }
$$

In view of (18), we have the following stationary conditions:

$$
\begin{gathered}
1-\left.\eta(s)^{(\alpha)}\right|_{s=x}=0,\left.\quad \eta(s)\right|_{s=x}=0, \\
\left.\eta(s)^{(2 \alpha)}\right|_{s=x}=0 .
\end{gathered}
$$

So, from (19), we get

$$
\eta(s)=\frac{(s-x)^{\alpha}}{\Gamma(1+\alpha)} .
$$

The initial value $u_{0}(x)$ is given by

$$
u_{0}(x)=u(0)+\frac{x^{\alpha}}{\Gamma(1+\alpha)} u^{(\alpha)}(0) .
$$

In view of (20), we have

$$
\begin{aligned}
u_{n+1}(x)= & u_{n}(x)+{ }_{0} I_{x}^{(\alpha)} \frac{(s-x)^{\alpha}}{\Gamma(1+\alpha)} \\
& \times\left\{L_{\alpha} u_{n}(s)+R_{\alpha} \widetilde{u}_{n}(s)-\tilde{g}(s)\right\} .
\end{aligned}
$$

Finally, from (22), we obtain the solution of (14) as follows:

$$
u=\lim _{n \rightarrow \infty} u_{n}
$$

\section{Local Fractional Series Expansion Method for the Helmholtz Equation}

Let us consider the following Helmholtz equation involving local fractional derivative operators:

$$
\frac{\partial^{2 \alpha} u(x, y)}{\partial x^{2 \alpha}}+\frac{\partial^{2 \alpha} u(x, y)}{\partial y^{2 \alpha}}=u(x, y)
$$

We now present the initial value conditions as follows:

$$
\begin{gathered}
u(0, y)=0, \\
\frac{\partial}{\partial x^{\alpha}} u(0, y)=E_{\alpha}\left(y^{\alpha}\right) .
\end{gathered}
$$

Using relation (12), we have

$$
\begin{gathered}
u_{i+2}(y)=\left(L_{\alpha} u_{i}\right)(y), \\
u_{0}(y)=u(0, y)=0, \\
u_{1}(y)=\frac{\partial}{\partial x^{\alpha}} u(0, y)=E_{\alpha}\left(y^{\alpha}\right),
\end{gathered}
$$

where

$$
L_{\alpha} u_{i}=u_{i}-\frac{\partial^{2 \alpha} u_{i}}{\partial y^{2 \alpha}}
$$

Hence, we get the following iterative relations:

$$
\begin{gathered}
u_{i+2}(y)=\left(u_{i}-\frac{\partial^{2 \alpha} u_{i}}{\partial y^{2 \alpha}}\right)(y), \\
u_{0}(y)=u(0, y)=0, \\
u_{i+2}(y)=\left(u_{i}-\frac{\partial^{2 \alpha} u_{i}}{\partial y^{2 \alpha}}\right)(y), \\
u_{1}(y)=E_{\alpha}\left(y^{\alpha}\right) .
\end{gathered}
$$

From (28), we have

$$
u_{0}(y)=u_{2}(y)=u_{4}(y)=\cdots=0
$$

From (29), we get the following terms:

$$
\begin{aligned}
u_{1}(y) & =E_{\alpha}\left(y^{\alpha}\right), \\
u_{3}(y) & =\left(u_{1}-\frac{\partial^{2 \alpha} u_{1}}{\partial y^{2 \alpha}}\right)(y) \\
& =\left[E_{\alpha}\left(y^{\alpha}\right)-E_{\alpha}\left(y^{\alpha}\right)\right]=0,
\end{aligned}
$$




$$
\begin{aligned}
& u_{5}(y)=0, \\
& u_{7}(y)=\cdots=0 .
\end{aligned}
$$

Hence, we obtain

$$
u(x, y)=\frac{x^{\alpha}}{\Gamma(1+\alpha)} E_{\alpha}\left(y^{\alpha}\right)
$$

\section{Local Fractional Variational Iteration Method for the Helmholtz Equation}

We now consider (24) with the initial and boundary conditions in (25) by using the local fractional variational iteration method.

Applying the iterative relation equation (22), we get

$$
\begin{aligned}
u_{n+1}(x, y)= & u_{n}(x, y)+{ }_{0} I_{y}^{(\alpha)} \frac{(s-y)^{\alpha}}{\Gamma(1+\alpha)} \\
& \times\left\{\frac{\partial^{2 \alpha} u_{n}(x, y)}{\partial x^{2 \alpha}}+\frac{\partial^{2 \alpha} u_{n}(x, y)}{\partial y^{2 \alpha}}-u_{n}(x, y)\right\},
\end{aligned}
$$

where the initial value is given by

$$
u_{0}(x, y)=\frac{x^{\alpha}}{\Gamma(1+\alpha)} E_{\alpha}\left(y^{\alpha}\right)
$$

Therefore, from (34) we have

$$
\begin{aligned}
u_{1}(x, y)= & u_{0}(x, y)+{ }_{0} I_{y}^{(\alpha)} \frac{(s-y)^{\alpha}}{\Gamma(1+\alpha)} \\
& \times\left\{\frac{\partial^{2 \alpha} u_{0}(x, y)}{\partial x^{2 \alpha}}+\frac{\partial^{2 \alpha} u_{0}(x, y)}{\partial y^{2 \alpha}}-u_{0}(x, y)\right\} \\
= & \frac{x^{\alpha}}{\Gamma(1+\alpha)} E_{\alpha}\left(y^{\alpha}\right) .
\end{aligned}
$$

The second approximate term reads as follows:

$$
\begin{aligned}
u_{2}(x, y)= & u_{1}(x, y)+{ }_{0} I_{y}^{(\alpha)} \frac{(s-y)^{\alpha}}{\Gamma(1+\alpha)} \\
& \times\left\{\frac{\partial^{2 \alpha} u_{1}(x, y)}{\partial x^{2 \alpha}}+\frac{\partial^{2 \alpha} u_{1}(x, y)}{\partial y^{2 \alpha}}-u_{1}(x, y)\right\} \\
= & \frac{x^{\alpha}}{\Gamma(1+\alpha)} E_{\alpha}\left(y^{\alpha}\right) .
\end{aligned}
$$

The third approximate term reads as follows:

$$
\begin{aligned}
u_{3}(x, y)= & u_{2}(x, y)+{ }_{0} I_{y}^{(\alpha)} \frac{(s-y)^{\alpha}}{\Gamma(1+\alpha)} \\
& \times\left\{\frac{\partial^{2 \alpha} u_{2}(x, y)}{\partial x^{2 \alpha}}+\frac{\partial^{2 \alpha} u_{2}(x, y)}{\partial y^{2 \alpha}}-u_{2}(x, y)\right\} \\
= & \frac{x^{\alpha}}{\Gamma(1+\alpha)} E_{\alpha}\left(y^{\alpha}\right) .
\end{aligned}
$$

Other approximate terms are presented as follows:

$$
\begin{aligned}
& u_{4}(x, y)=u_{3}(x, y)+{ }_{0} I_{y}^{(\alpha)} \frac{(s-y)^{\alpha}}{\Gamma(1+\alpha)} \\
& \times\left\{\frac{\partial^{2 \alpha} u_{3}(x, y)}{\partial x^{2 \alpha}}+\frac{\partial^{2 \alpha} u_{3}(x, y)}{\partial y^{2 \alpha}}-u_{3}(x, y)\right\} \\
& =\frac{x^{\alpha}}{\Gamma(1+\alpha)} E_{\alpha}\left(y^{\alpha}\right) \text {, } \\
& u_{5}(x, y)=u_{4}(x, y)+{ }_{0} I_{y}^{(\alpha)} \frac{(s-y)^{\alpha}}{\Gamma(1+\alpha)} \\
& \times\left\{\frac{\partial^{2 \alpha} u_{4}(x, y)}{\partial x^{2 \alpha}}+\frac{\partial^{2 \alpha} u_{4}(x, y)}{\partial y^{2 \alpha}}-u_{4}(x, y)\right\} \\
& =\frac{x^{\alpha}}{\Gamma(1+\alpha)} E_{\alpha}\left(y^{\alpha}\right) \\
& u_{n}(x, y)=u_{n-1}(x, y)+{ }_{0} I_{y}^{(\alpha)} \frac{(s-y)^{\alpha}}{\Gamma(1+\alpha)} \\
& \times\left\{\frac{\partial^{2 \alpha} u_{n-1}(x, y)}{\partial x^{2 \alpha}}+\frac{\partial^{2 \alpha} u_{n-1}(x, y)}{\partial y^{2 \alpha}}\right. \\
& \left.-u_{n-1}(x, y)\right\} \\
& =\frac{x^{\alpha}}{\Gamma(1+\alpha)} E_{\alpha}\left(y^{\alpha}\right)
\end{aligned}
$$

and so on.

So, we get

$$
u(x, y)=\lim _{n \rightarrow \infty} u_{n}(x, y)=\frac{x^{\alpha}}{\Gamma(1+\alpha)} E_{\alpha}\left(y^{\alpha}\right) .
$$

The result is the same as the one which is obtained by the local fractional series expansion method. The nondifferentiable solution is shown in Figure 1.

\section{Conclusions}

In this work, the nondifferentiable solution for the Helmholtz equation involving local fractional derivative operators is 


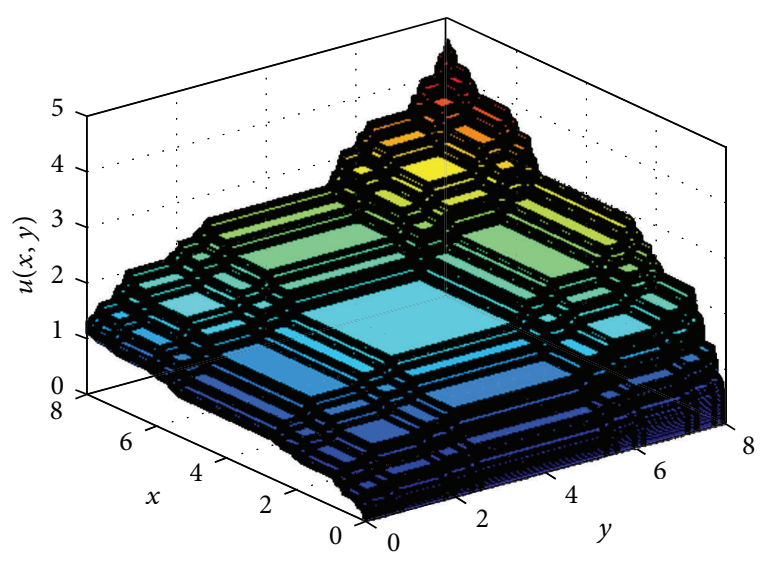

Figure 1: Graph of $u(x, y)$ for $\alpha=\ln 2 / \ln 3$.

investigated by using the local fractional series expansion method and the variational iteration method. By using these two markedly different methods, the same solution is obtained. These two approaches are remarkably efficient to process other linear local fractional differential equations as well.

\section{Conflict of Interests}

The authors declare that there is no conflict of interests regarding the publication of this paper.

\section{Acknowledgments}

This work was supported by the National Scientific and Technological Support Projects (no. 2012BAE09B00), the National Natural Science Foundation of China (nos. 11126213 and 61170317), and the National Natural Science Foundation of the Hebei Province (nos. A2012209043 and E2013209215).

\section{References}

[1] R. Kreß and G. F. Roach, "Transmission problems for the Helmholtz equation," Journal of Mathematical Physics, vol. 19, no. 6, pp. 1433-1437, 1978.

[2] A. Deraemaeker, I. Babuška, and P. Bouillard, "Dispersion and pollution of the FEM solution for the helmholtz equation in one, two and three dimensions," International Journal for Numerical Methods in Engineering, vol. 46, no. 4, pp. 471-499, 1999.

[3] A. Hannukainen, M. Huber, and J. Schöberl, "A mixed hybrid finite element method for the Helmholtz equation," Journal of Modern Optics, vol. 58, no. 5-6, pp. 424-437, 2011.

[4] S. Momani and S. Abuasad, "Application of He's variational iteration method to Helmholtz equation," Chaos, Solitons \& Fractals, vol. 27, no. 5, pp. 1119-1123, 2006.

[5] M. Rafei and D. D. Ganji, "Explicit solutions of Helmholtz equation and fifth-order KdV equation using homotopy perturbation method," International Journal of Nonlinear Sciences and Numerical Simulation, vol. 7, no. 3, pp. 321-328, 2006.

[6] J.-D. Benamou and B. Desprès, "A domain decomposition method for the Helmholtz equation and related optimal control problems," Journal of Computational Physics, vol. 136, no. 1, pp. 68-82, 1997.

[7] M. M. Grigoriev and G. F. Dargush, "A fast multi-level boundary element method for the Helmholtz equation," Computer Methods in Applied Mechanics and Engineering, vol. 193, no. 3-5, pp. 165-203, 2004.

[8] S. Tomioka, S. Nisiyama, M. Itagaki, and T. Enoto, "Internal field error reduction in boundary element analysis for Helmholtz equation," Engineering Analysis with Boundary Elements, vol. 23, no. 3, pp. 211-222, 1999.

[9] O. F. Næss and K. S. Eckhoff, "A modified Fourier-Galerkin method for the Poisson and Helmholtz equations," Journal of Scientific Computing, vol. 17, no. 1-4, pp. 529-539, 2002.

[10] C. M. Linton, “The Green's function for the two-dimensional Helmholtz equation in periodic domains," Journal of Engineering Mathematics, vol. 33, no. 4, pp. 377-401, 1998.

[11] A. Dienstfrey, F. Hang, and J. Huang, "Lattice sums and the two-dimensional, periodic Green's function for the Helmholtz equation," Proceedings of the Royal Society A, vol. 457, no. 2005, pp. 67-85, 2001.

[12] J. T. Machado, V. Kiryakova, and F. Mainardi, "Recent history of fractional calculus," Communications in Nonlinear Science and Numerical Simulation, vol. 16, no. 3, pp. 1140-1153, 2011.

[13] I. Podlubny, Fractional Differential Equations, vol. 198 of Mathematics in Science and Engineering, Academic Press, San Diego, Calif, USA, 1999.

[14] B. J. West, M. Bologna, and P. Grigolini, Physics of Fractal Operators, Institute for Nonlinear Science, Springer, New York, NY, USA, 2003.

[15] A. A. Kilbas, H. M. Srivastava, and J. J. Trujillo, Theory and Applications of Fractional Differential Equations, vol. 204 of North-Holland Mathematics Studies, Elsevier Science, Amsterdam, The Netherlands, 2006.

[16] R. L. Magin, Fractional Calculus in Bioengineering, Begerll House, West Redding, Conn, USA, 2006.

[17] J. Sabatier, O. P. Agrawal, and J. A. T. Machado, Advances in Fractional Calculus: Theoretical Developments and Applications in Physics and Engineering, Springer, New York, NY, USA, 2007.

[18] G. M. Zaslavsky, Hamiltonian Chaos and Fractional Dynamics, Oxford University Press, Oxford, UK, 2008.

[19] F. Mainardi, Fractional Calculus and Waves in Linear Viscoelasticity: An Introduction to Mathematical Models, World Scientific Publishing, Singapore, 2010.

[20] R. Herrmann, Fractional Calculus: An Introduction for Physicists, World Scientific Publishing, Singapore, 2011.

[21] J. Klafter, S. C. Lim, and R. Metzler, Fractional Dynamics: Recent Advances, World Scientific Publishing, Singapore, 2012.

[22] S. Das, Functional Fractional Calculus, Springer, Berlin, Germany, 2nd edition, 2011.

[23] V. E. Tarasov, Fractional Dynamics: Applications of Fractional Calculus to Dynamics of Particles, Fields and Media, Springer, Berlin, Germany, 2011.

[24] A. B. Malinowska and D. F. M. Torres, Introduction to the Fractional Calculus of Variations, Imperial College Press, London, UK, 2012.

[25] H. Sheng, Y. Chen, and T. Qiu, Fractional Processes and Fractional-Order Signal Processing: Techniques and Applications, Signals and Communication Technology, Springer, New York, NY, USA, 2012.

[26] D. Baleanu, J. A. T. Machado, and A. C. Luo, Fractional Dynamics and Control, Springer, New York, NY, USA, 2012. 
[27] E. Goldfain, "Fractional dynamics, Cantorian space-time and the gauge hierarchy problem," Chaos, Solitons \& Fractals, vol. 22, no. 3, pp. 513-520, 2004.

[28] M. S. Samuel and A. Thomas, "On fractional Helmholtz equations," Fractional Calculus \& Applied Analysis, vol. 13, no. 3, pp. 295-308, 2010.

[29] P. K. Gupta, A. Yildirim, and K. N. Rai, "Application of He's homotopy perturbation method for multi-dimensional fractional Helmholtz equation," International Journal of Numerical Methods for Heat \& Fluid Flow, vol. 22, no. 3-4, pp. 424-435, 2012.

[30] K. M. Kolwankar and A. D. Gangal, "Local fractional FokkerPlanck equation," Physical Review Letters, vol. 80, no. 2, pp. 214217, 1998.

[31] A. Carpinteri, B. Chiaia, and P. Cornetti, "On the mechanics of quasi-brittle materials with a fractal microstructure," Engineering Fracture Mechanics, vol. 70, no. 16, pp. 2321-2349, 2003.

[32] F. Ben Adda and J. Cresson, "About non-differentiable functions," Journal of Mathematical Analysis and Applications, vol. 263, no. 2, pp. 721-737, 2001.

[33] A. Babakhani and V. Daftardar-Gejji, "On calculus of local fractional derivatives," Journal of Mathematical Analysis and Applications, vol. 270, no. 1, pp. 66-79, 2002.

[34] Y. Chen, Y. Yan, and K. Zhang, "On the local fractional derivative," Journal of Mathematical Analysis and Applications, vol. 362, no. 1, pp. 17-33, 2010.

[35] W. Chen, H. Sun, X. Zhang, and D. Korošak, "Anomalous diffusion modeling by fractal and fractional derivatives," Computers \& Mathematics with Applications, vol. 59, no. 5, pp. 1754-1758, 2010.

[36] X.-J. Yang, Local Fractional Functional Analysis and Its Applications, Asian Academic Publisher, Hong Kong, 2011.

[37] X.-J. Yang, Advanced Local Fractional Calculus and Its Applications, World Science Publisher, New York, NY, USA, 2012.

[38] A. K. Golmankhaneh, V. Fazlollahi, and D. Baleanu, "Newtonian mechanics on fractals subset of real-line," Romania Reports in Physics, vol. 65, pp. 84-93, 2013.

[39] Y.-J. Hao, H. M. Srivastava, H. Jafari, and X.-J. Yang, "Helmholtz and diffusion equations associated with local fractional derivative operators involving the Cantorian and Cantor-type cylindrical coordinates," Advances in Mathematical Physics, vol. 2013, Article ID 754248, 5 pages, 2013.

[40] A.-M. Yang, X.-J. Yang, and Z.-B. Li, "Local fractional series expansion method for solving wave and diffusion equations on Cantor sets," Abstract and Applied Analysis, vol. 2013, Article ID 351057, 5 pages, 2013.

[41] X.-J. Yang and D. Baleanu, "Fractal heat conduction problem solved by local fractional variation iteration method," Thermal Science, vol. 17, no. 2, pp. 625-628, 2013.

[42] W.-H. Su, D. Baleanu, X.-J. Yang, and H. Jafari, "Damped wave equation and dissipative wave equation in fractal strings within the local fractional variational iteration method," Fixed Point Theory and Applications, vol. 2013, no. 1, article 89, pp. 1-11, 2013.

[43] Y.-J. Yang, D. Baleanu, and X.-J. Yang, "A local fractional variational iteration method for Laplace equation within local fractional operators," Abstract and Applied Analysis, vol. 2013, Article ID 202650, 6 pages, 2013.

[44] J.-H. He, "Local fractional variational iteration method for fractal heat transfer in silk cocoon hierarchy," Nonlinear Science Letters A, vol. 4, no. 1, pp. 15-20, 2013. 


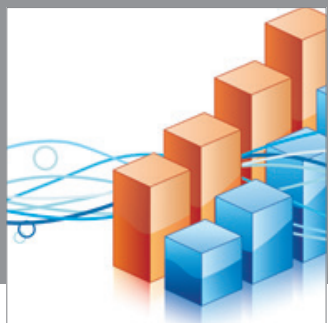

Advances in

Operations Research

mansans

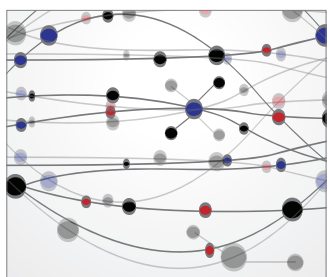

The Scientific World Journal
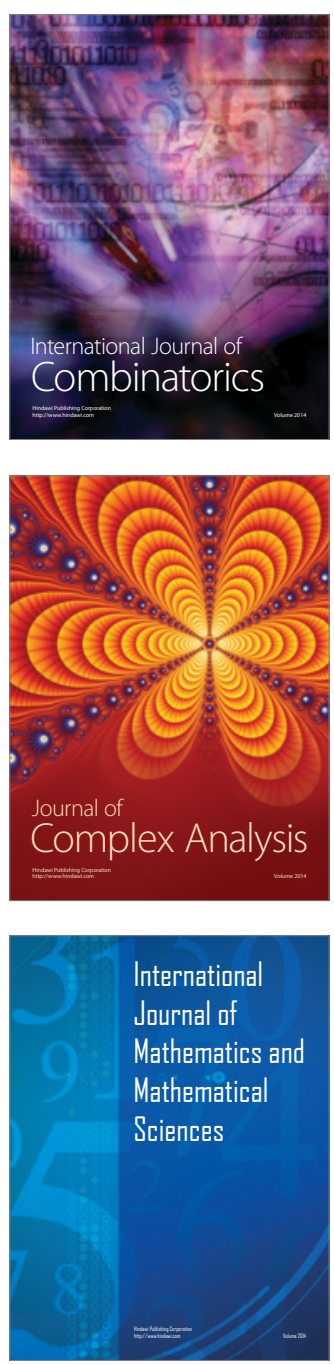
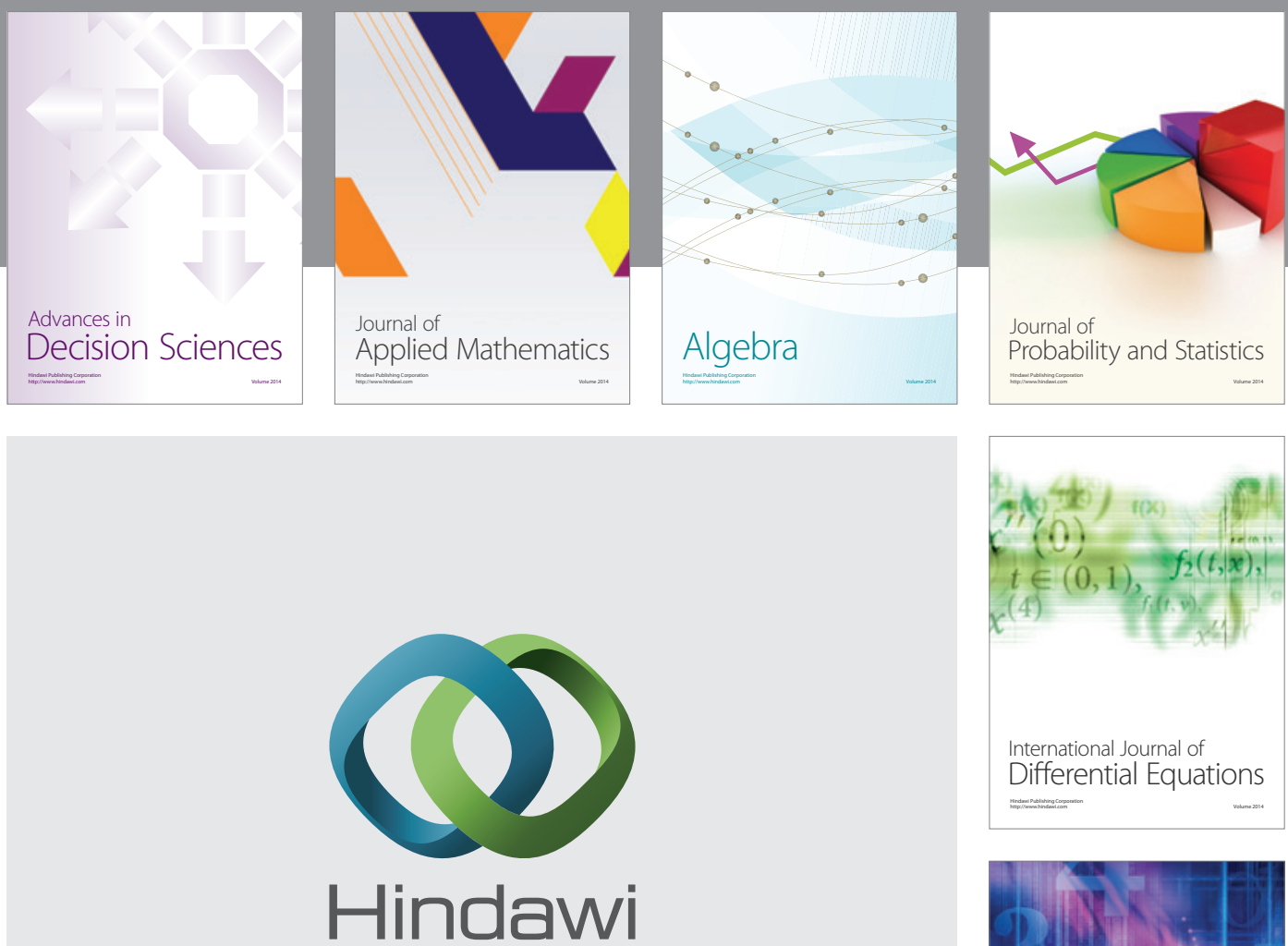

Submit your manuscripts at http://www.hindawi.com
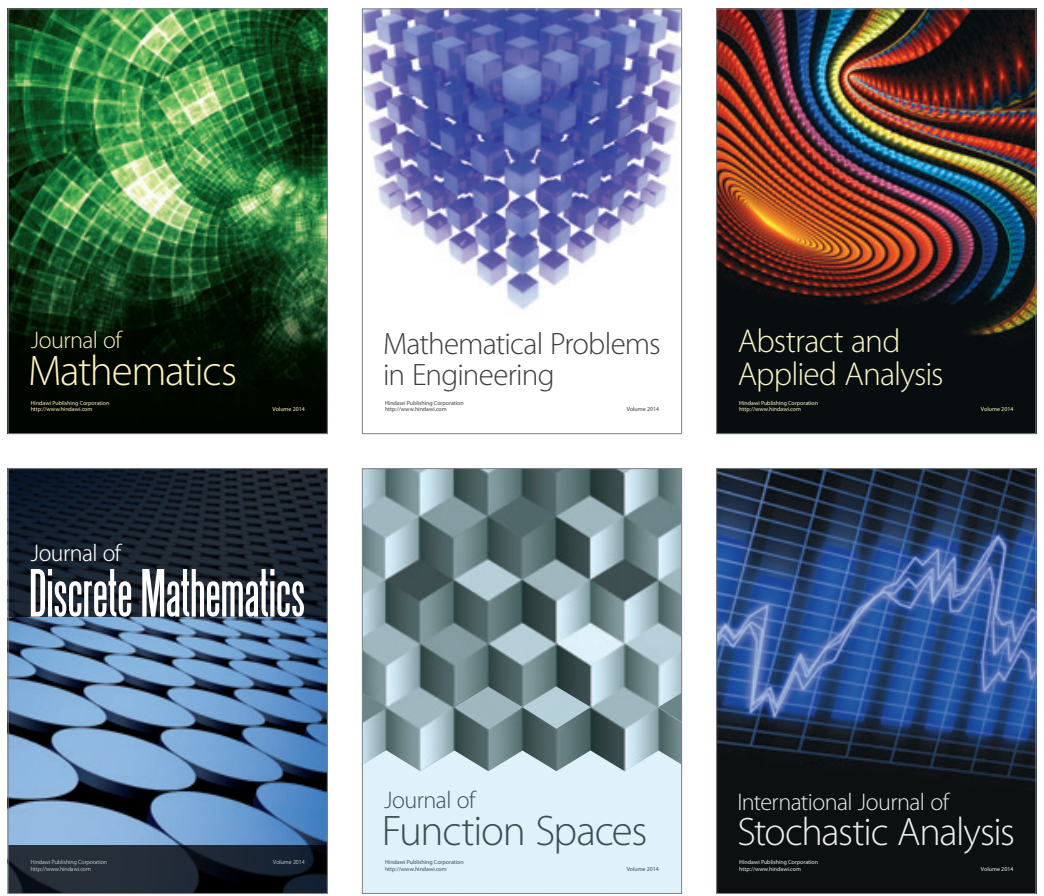

Journal of

Function Spaces

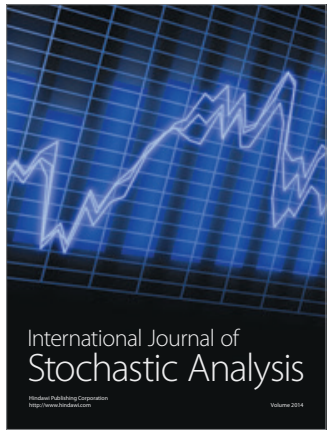

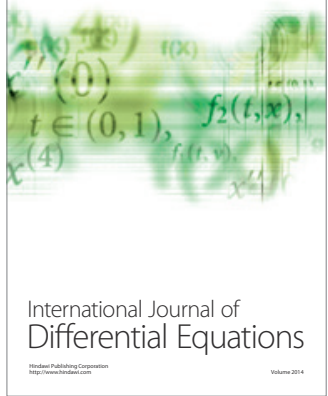
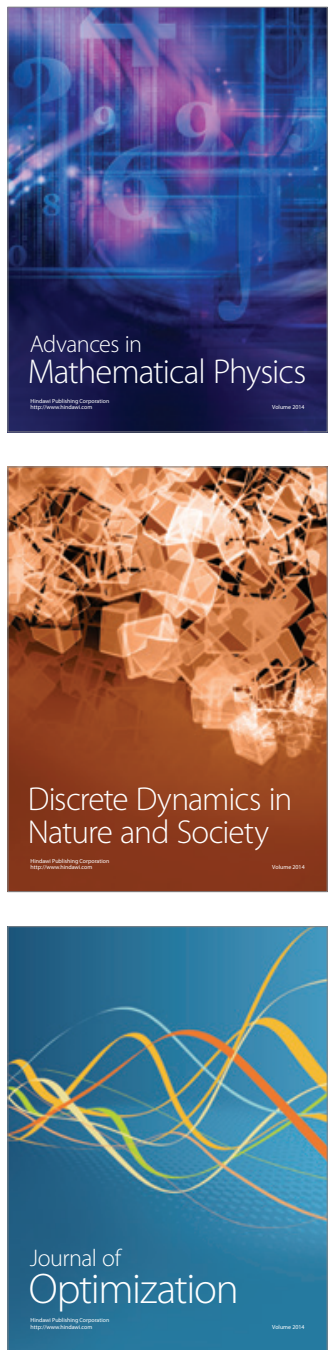